\title{
三聚氯氰促进的咪唑并[1,2-a]吡啶类化合物的合成
}

\author{
尹志奎 ${ }^{a}$ 田拴宝 ${ }^{*, b}$ \\ ( ${ }^{a}$ 新乡医学院药学院 新乡 453003) \\ ( ${ }^{b}$ 新乡医学院基础医学院 新乡 453003)
}

\begin{abstract}
摘要 以三聚氯氰作为反应促进剂, 在室温和 $\mathrm{CH}_{3} \mathrm{CN}$ 作为溶剂的条件下, 利用取代 2-氨基吡啶、醛和烷基异腈三组分 缩合, 高收率地实现了一系列咪唑并 $[1,2-a]$ 吡啶类化合物的合成. 产物结构经 ${ }^{1} \mathrm{H} \mathrm{NMR},{ }^{13} \mathrm{C} N M R$ 和元素分析等进行了 表征.

关键词＼cjkstart咪唑并 $[1,2-a]$ 吡啶类化合物; 三聚氯氧; Groebcke 反应; 多组分反应; 合成
\end{abstract}

\section{Cyanuric Chloride-Promoted Synthesis of Imidazo[1,2-a]pyridines}

\author{
Yin, Zhikui $^{a} \quad$ Tian, Shuanbao ${ }^{*, b}$ \\ ( ${ }^{a}$ School of Pharmacy, Xinxiang Medical University, Xinxiang 453003) \\ ( ${ }^{b}$ School of Basic Medical Sciences, Xinxiang Medical University, Xinxiang 453003)
}

\begin{abstract}
Cyanuric chloride-promoted synthesis of imidazo[1,2-a]pyridine derivatives was realized in high yields by the reaction of 2-aminopyridines, aldehydes and isonitriles in $\mathrm{CH}_{3} \mathrm{CN}$ at room temperature. All products were characterized by ${ }^{1} \mathrm{H}$ NMR, ${ }^{13} \mathrm{C}$ NMR and elemental analysis.

Keywords Imidazo[1,2-a]pyridines; cyanuric chloride; Groebcke reaction; multi-component reaction; synthesis
\end{abstract}

咪唑并 $[1,2-a]$ 吡啶是一种常见的含氮稠杂环. 它的 衍生物具有抗肿瘤 ${ }^{[1]}$ 、抗病毒 ${ }^{[2]}$ 、抗溃疡 ${ }^{[3]}$ 、镇静催眠 ${ }^{[4]}$ 和抗寄生虫 ${ }^{[5]}$ 等多种生物活性, 许多药物如奥普力农、 阿吡坦、Zolipidem 都含有咪唑并[1,2- $a$ ]吡啶的结构单元. 目前有关咪唑并 $[1,2-a]$ 吡啶衍生物合成和生物活性的报 道与日俱增 ${ }^{[6]}$. 其中, 利用 Groebcke 反应三组分缩合取 代 2-氨基吡啶、醛和烷基异腈可有效的合成这类化合物. 通常, Groebcke 反应需在路易斯酸 ${ }^{[7]}$ 或质子酸 ${ }^{88}$ 催化下 进行, 然而, 在使用这些催化剂中存在一些问题, 如使 用过量的催化剂、较长的反应时间、剧烈的反应条件等. 为了克服这些问题, 近年来, 利用微波技术 ${ }^{[9]}$ 和离子液 体 ${ }^{[10]}$ 合成咪唑并 $[1,2-a]$ 吡啶类化合物的 Groebcke 反应 也已有文献报道.

三聚氯氰(TCT) 是一种价格便宜, 易处理的试剂. 近年来国内外许多学者发现 TCT 可作为催化剂或促进 剂来改善反应条件, 促进合成反应和提高反应收率等. 一些化合物如 2-芳基-2,3-二氢喹啉-4(1H)-酮类化合 物 ${ }^{[1]}$ 、双弜哚甲烷类化合物 ${ }^{[12]} 、 N$-磺酰亚胺类化合物 ${ }^{[13]}$ 和 14-烷基或烷基-14H-二苯并 $[a, j]$ 氧杂葱酮类化合物 ${ }^{[14]}$
和 1,5-苯并硫氮杂类化合物 ${ }^{[15]}$ 已被用 TCT 作为催化剂 或促进剂成功合成.

为了研究咪唑并 $[1,2-a]$ 吡啶类化合物的生物活性, 我们对其合成进行了研究. 以 TCT 作为反应促进剂, 在 室温和 $\mathrm{CH}_{3} \mathrm{CN}$ 作为溶剂的条件下, 通过取代 2-氨基吡 啶、醛和烷基异腈三组分缩合，高收率地实现了一系列 咪唑并 $[1,2-a]$ 吡啶类化合物的合成(Eq. 1 和表 1).

$$
\text { R }
$$

\section{1 结果与讨论}

\section{1 反应条件的探讨}

首先, 在室温和 $\mathrm{CH}_{3} \mathrm{CN}$ 作为溶剂条件下, 我们以

* E-mail: tianshuanbao2006@163.com

Received July 3, 2013; revised September 23, 2013; published online October 11, 2013. 
表 1 合成咪唑并 $[1,2-a$ 吡啶类化合物

Table 1 Synthesis of imidazo[1,2-a]pyridine derivatives

\begin{tabular}{|c|c|c|c|c|c|c|}
\hline Entry & $\mathrm{R}^{1}$ & $\mathrm{R}^{2}$ & $\mathrm{R}^{3}$ & Product & Time/h & Yield/\% \\
\hline 1 & $\mathrm{H}$ & $\mathrm{C}_{6} \mathrm{H}_{5}$ & $c$-Hex & $4 a$ & 1.0 & 93 \\
\hline 2 & $\mathrm{H}$ & 4-Cl- $\mathrm{C}_{6} \mathrm{H}_{4}$ & $t-\mathrm{Bu}$ & $4 b$ & 1.0 & 92 \\
\hline 3 & $\mathrm{H}$ & $4-\mathrm{Cl}-\mathrm{C}_{6} \mathrm{H}_{4}$ & $c$-Hex & $4 c$ & 1.0 & 92 \\
\hline 4 & $\mathrm{H}$ & $4-\mathrm{Br}-\mathrm{C}_{6} \mathrm{H}_{4}$ & $t-\mathrm{Bu}$ & $4 d$ & 1.0 & 90 \\
\hline 5 & $\mathrm{H}$ & $4-\mathrm{NO}_{2}-\mathrm{C}_{6} \mathrm{H}_{4}$ & $c$-Hex & $4 e$ & 1.0 & 95 \\
\hline 6 & $\mathrm{H}$ & $3-\mathrm{HO}-\mathrm{C}_{6} \mathrm{H}_{4}$ & $t-\mathrm{Bu}$ & $4 f$ & 1.5 & 87 \\
\hline 7 & $\mathrm{H}$ & 2-Furyl & $c$-Hex & $4 g$ & 1.0 & 88 \\
\hline 8 & $3-\mathrm{CH}_{3}$ & 4-Cl- $\mathrm{C}_{6} \mathrm{H}_{4}$ & $c$-Hex & $4 h$ & 1.0 & 91 \\
\hline 9 & $5-\mathrm{CH}_{3}$ & $\mathrm{C}_{6} \mathrm{H}_{5}$ & $t-\mathrm{Bu}$ & $4 i$ & 1.5 & 89 \\
\hline 10 & $5-\mathrm{CH}_{3}$ & $3,4,5-(\mathrm{MeO})_{3}-\mathrm{C}_{6} \mathrm{H}_{2}$ & $t-\mathrm{Bu}$ & $4 j$ & 1.0 & 93 \\
\hline 11 & $5-\mathrm{CH}_{3}$ & $4-\mathrm{NO}_{2}-\mathrm{C}_{6} \mathrm{H}_{4}$ & $t-\mathrm{Bu}$ & $4 k$ & 1.0 & 96 \\
\hline 12 & $5-\mathrm{CH}_{3}$ & $4-\mathrm{Br}-\mathrm{C}_{6} \mathrm{H}_{4}$ & $t-\mathrm{Bu}$ & 41 & 1.5 & 88 \\
\hline 13 & $5-\mathrm{CH}_{3}$ & 2-Thienyl & $c$-Hex & $4 m$ & 1.5 & 89 \\
\hline
\end{tabular}

2-氨基吡啶、苯甲醛和叔丁基异腈反应生成 $N$-叔丁基-2苯基咪唑并 $[1,2-a]$ 吡啶-3-胺(4a)作为典型反应，考查了 不同催化剂或促进剂对 4a 产率的影响. 从表 2 可以看 出: 在无催化剂或促进剂条件下即使反应 $12 \mathrm{~h}$ 也只有少 量的目标产物生成, TCT 和其他催化剂或促进剂相比具 有反应时间短和产率高的特点. 接下来, 对 TCT 的用量 进行了进一步考查, 发现用 $2 \mathrm{~mol} \%$ 的 TCT 得到较高产 率. 当用 $1 \mathrm{~mol} \%$ 的 TCT 在 $2 \mathrm{~h}$ 时观测产率较低, 而用 3 mol\%或更大量的 TCT, $\mathbf{4 a}$ 的产率并没有明显提高. 在室

表 2 反应条件的优化 ${ }^{a}$

Table 2 Optimization of the reaction conditions

\begin{tabular}{clccc}
\hline Entry & \multicolumn{1}{c}{ Catalyst } & $\begin{array}{c}\text { Amount of } \\
\text { catalyst/mmol }\end{array}$ & Time/h & Yield/\% \\
\hline 1 & No & 0 & 12 & Trace \\
2 & $p$-TsOH & 0.2 & 2.0 & 63 \\
3 & $\mathrm{H}_{2} \mathrm{SO}_{4}$ & 0.2 & 2.0 & 56 \\
4 & Sulfamic acid $_{5}$ & 0.2 & 2.0 & 65 \\
5 & $\mathrm{ZrOCl}_{2} \bullet 8 \mathrm{H}_{2} \mathrm{O}$ & 0.2 & 1.0 & 69 \\
6 & $\mathrm{SbCl}_{3}$ & 0.2 & 1.5 & 48 \\
7 & $\mathrm{TCT}$ & 0.1 & 2.0 & 69 \\
8 & $\mathrm{TCT}$ & 0.2 & 1.0 & 93 \\
9 & $\mathrm{TCT}$ & 0.3 & 1.0 & 92 \\
10 & $\mathrm{TCT}$ & 0.4 & 1.0 & 92 \\
11 & TCT & 0.5 & 1.0 & 93 \\
\hline
\end{tabular}

a 2 -氨基吡啶 $(10 \mathrm{mmol})$, 苯甲醛 $(10 \mathrm{mmol})$, 叔丁基异腈 $(10 \mathrm{mmol}), \mathrm{CH}_{3} \mathrm{CN}$ (30 mL), 室温.
温和 $\mathrm{CH}_{3} \mathrm{CN}$ 作为溶剂的条件下，用 $2 \mathrm{~mol} \% \mathrm{TCT}$ 作为反 应促进剂是最佳反应条件.

\section{2 反应普遍性的探讨}

为了研究此反应的适用范围, 我们用一系列带有不 同取代基的芳醛和取代 2-氨基吡啶、烷基异腈反应. 实 验结果表明: 不管是连有吸电子的芳醛，还是连有供电 子的芳醛，都得到了预期的产物(表 1, Entries $1 \sim 6,8 \sim$ 12). 为了拓宽反应的范围, 我们把该方法延伸到三组 分合 2-醛基噻吩或糠醛、2-氨基吡啶和烷基异腈，也得 到了预期的产物(表 1, Entries 7,13), 所有产物结构经 ${ }^{1} \mathrm{H}$ NMR, ${ }^{13} \mathrm{C}$ NMR 和元素分析等进行了表征.

\section{3 反应有效性的探讨}

为了证明该反应的有效性，在表 3 中，我们以 2-氨 基吡啶、苯甲醛和环己基异腈反应生成 $N$-环己基-2-苯 基咪唑并 $[1,2-a]$ 吡啶-3-胺(4b)作为典型反应，比较了该 方法与其他方法的优略之处, 从表 3 可以看出，该方法 具有反应条件温和、产率高的优点.

\section{4 反应机理的研究}

三聚氯氰化学性质非常活泼，遇水及碱易分解成三 聚氰酸，同时放出的氯化氢气体. 由此，推断本合成方 法的机理如 Scheme 1. 首先醛与取代 2-氨基吡啶反应生 成亚胺 I 与水, 生成的水分子与三聚氯氧反应形成 3

表 3 不同的催化体系对 $\mathbf{4 b}$ 产率的影响 ${ }^{a}$

Table 3 Yield of product $\mathbf{4 b}$ effected different catalytic system

\begin{tabular}{clrrr}
\hline Entry & \multicolumn{1}{c}{ Reaction condition } & Time $/$ min & Yield/\% & Ref. \\
\hline 1 & $\mathrm{ZnCl}_{2}(0.5 \mathrm{mmol}), 1,4$-dioxane, microwave & 60 & 62 & {$[7 \mathrm{~h}]$} \\
2 & $p$-TsOH $(0.52 \mathrm{mmol}), \mathrm{MeOH}$, r.t. & 120 & 94 & {$[8 \mathrm{e}]$} \\
3 & Montmorillonite $(188 \mathrm{mg}), 1,4$-dioxane, microwave, $100{ }^{\circ} \mathrm{C}$ & 30 & 78 & {$[7 \mathrm{i}]$} \\
4 & $4 \mathrm{~mol} / \mathrm{L} \mathrm{HCl} /$ dioxane $(4 \mu \mathrm{L}), \mathrm{CH}_{3} \mathrm{CN}$, microwave, $110{ }^{\circ} \mathrm{C}$ & 20 & 73 & {$[8 \mathrm{f}]$} \\
5 & $\mathrm{TCT}(0.2 \mathrm{mmol}), \mathrm{CH}_{3} \mathrm{CN}$, r.t. & 60 & 92 & The study \\
\hline
\end{tabular}

${ }^{a} 2$-氨基吡啶 $(10 \mathrm{mmol})$, 苯甲醛 $(10 \mathrm{mmol})$, 环已基异腈 $(10 \mathrm{mmol})$. 
equiv. $\mathrm{HCl}$, 然后, 在 $\mathrm{HCl}$ 作用下, 亚胺经历质子化, 形 成中间体 II, 它随后与异腈反生[4+1]环加成形成中间 体 III, 最后中间体 III 经历 1,3 氢转移生成相应的目标 化合物 4. 为了验证此反应机理的合理性, 我们分别考 察了通入 $6 \mathrm{~mol} \% \mathrm{HCl}$ 和加入 $2 \mathrm{~mol} \%$ 三聚氯氧对于 $\mathbf{4 a}$ 产率的影响, 实验结果显示: 通入 $6 \mathrm{~mol} \% \mathrm{HCl}$ 时, $4 \mathrm{a}$ 产 率为 $77 \%$, 加入 $6 \mathrm{~mol} \%$ 三聚氯氰时, $\mathbf{4 a}$ 产率为 $93 \%$, 两 者具有相似的效果, 但通入 $6 \mathrm{~mol} \% \mathrm{HCl}$ 时, $4 \mathrm{a}$ 产率略低 于加入 $2 \mathrm{~mol} \%$ 三聚氯氧.
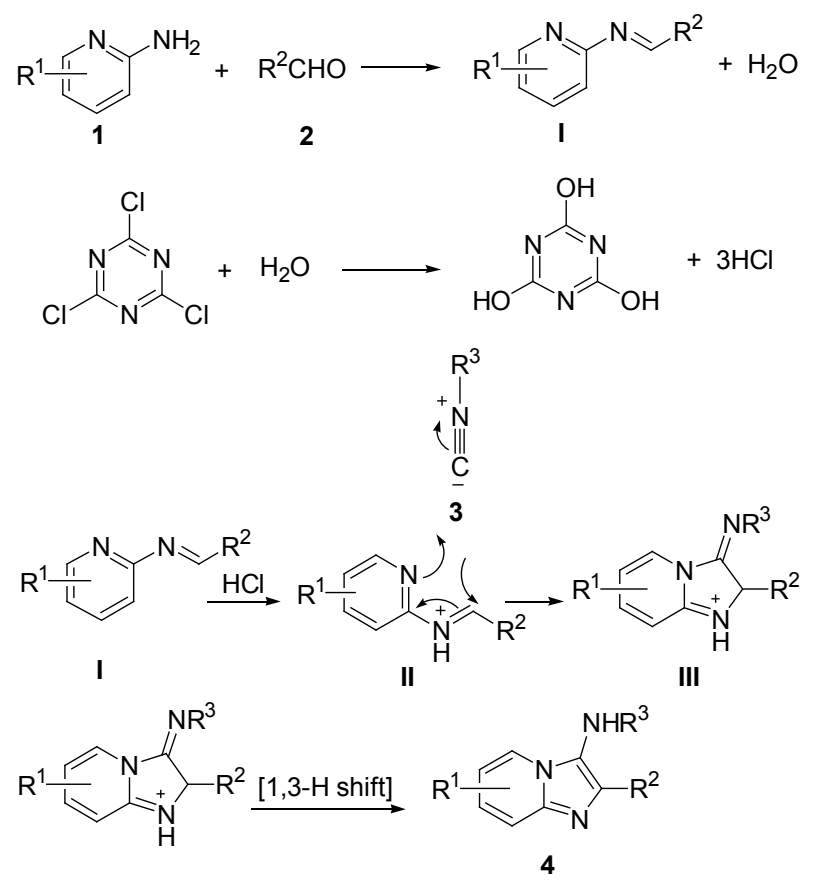

Scheme 1

\section{2 结论}

总之, 以 $\mathrm{TCT}$ 作为反应促进剂, 在室温和 $\mathrm{CH}_{3} \mathrm{CN}$ 作为溶剂的条件下, 通过取代 2-氨基吡啶、醛和烷基异 腈三组分缩合, 高收率地实现了一系列咪唑并 $[1,2-a]$ 吡 啶类化合物的合成. 此方法具有反应条件温和、操作简 单、反应时间短和产率高等优点, 它为咪唑并 $[1,2-a]$ 吡 啶类化合物的合成提供了一条新的途径.

\section{3 实验部分}

\section{1 仪器与试剂}

化合物熔点采用毛细管法测定, 温度计未校正; ${ }^{1} \mathrm{H}$ NMR, ${ }^{13} \mathrm{C}$ NMR 由 Bruker AV-400 核磁共振仪测定 (TMS 为内标, $\mathrm{CDCl}_{3}$ 为溶剂); 元素分析采用 Vario ELIII 元素分析仪测定. 所用试剂为市售化学纯或分析纯.

\section{2 咪唑并 $[1,2-a]$ 吡啶类化合物的合成}

将取代 2-氨基吡啶 $(10 \mathrm{mmol}) 、(10 \mathrm{mmol}) 、$ 基异腈
(10 mmol)和乙腈 $(30 \mathrm{~mL})$ 加到 $100 \mathrm{~mL}$ 三口瓶中, 摚拌下 加入 TCT $(0.2 \mathrm{mmol})$, 室温摚拌反应 $1 \sim 3 \mathrm{~h}$ 后, 冷却, 过滤, 蒸出溶剂, 粗产品用少量乙腈洗涤, 得化合物 4.

$N$-环己基-2-苯基咪唑并 $[1,2-a$ 吡啶-3-胺(4a)：黄色 固体. m.p. $198 \sim 200{ }^{\circ} \mathrm{C}$ (文献值 ${ }^{[8 g]} 200 \sim 202{ }^{\circ} \mathrm{C}$ ); ${ }^{1} \mathrm{H}$ NMR $\left(\mathrm{CDCl}_{3}, 400 \mathrm{MHz}\right) \delta: 1.00 \sim 1.69(\mathrm{~m}, 10 \mathrm{H}), 2.83 \sim$ $2.85(\mathrm{~m}, 1 \mathrm{H}), 4.42$ (brs, $1 \mathrm{H}), 6.58 \sim 6.63(\mathrm{~m}, 1 \mathrm{H}), 6.99 \sim$ $7.04(\mathrm{~m}, 1 \mathrm{H}), 7.19 \sim 8.01(\mathrm{~m}, 6 \mathrm{H}), 8.42 \sim 8.48(\mathrm{~m}, 1 \mathrm{H})$; ${ }^{13} \mathrm{C}$ NMR $\left(\mathrm{CDCl}_{3}, 100 \mathrm{MHz}\right) \delta: 24.7,25.4,33.9,56.8$, 112.2, 113.8, 115.0, 125.2, 127.02, 128.8, 128.9, 129.7, 136.0, 138.2, 142.7, 155.1. Anal. calcd for $\mathrm{C}_{19} \mathrm{H}_{21} \mathrm{~N}_{3}$ : C 78.32, H 7.26, N 14.42; found C 78.26, H 7.31, N 14.38.

$N$-叔丁基-2-(4-氯苯基)咪唑并 $[1,2-a]$ 吡啶-3-胺(4b): 褐色固体. m.p. 146 147 ${ }^{\circ} \mathrm{C}$ (文献值 ${ }^{[7]} 146{ }^{\circ} \mathrm{C}$ ); ${ }^{1} \mathrm{H}$ NMR $\left(\mathrm{CDCl}_{3}, 400 \mathrm{MHz}\right) \delta: 0.97$ (s, 9H), 3.0 (brs, 1H), $6.72(\mathrm{t}, J=7.0 \mathrm{~Hz}, 1 \mathrm{H}), 7.05(\mathrm{t}, J=7.2 \mathrm{~Hz}, 1 \mathrm{H}), 7.36(\mathrm{~d}$, $J=8.4 \mathrm{~Hz}, 2 \mathrm{H}), 7.49$ (d, $J=8.4 \mathrm{~Hz}, 1 \mathrm{H}), 7.88(\mathrm{~d}, J=8.4$ $\mathrm{Hz}, 2 \mathrm{H}), 8.15(\mathrm{~d}, J=7.0 \mathrm{~Hz}, 1 \mathrm{H}) ;{ }^{13} \mathrm{C}$ NMR $\left(\mathrm{CDCl}_{3}, 100\right.$ MHz) $\delta: 30.5,56.6,111.8,118.2,123.4,123.5,123.9$, 128.2, 129.7, 132.9, 134.0, 138.2, 142.5. Anal. calcd for $\mathrm{C}_{17} \mathrm{H}_{18} \mathrm{ClN}_{3}$ : C 68.11, H 6.05, N 14.02; found C 68.18, H 5.97, N 14.15 .

$N$-环已基-2-(4-氯苯基)咪唑并 $[1,2-a]$ 吡啶-3-胺(4c): 白色固体. m.p. $180 \sim 181{ }^{\circ} \mathrm{C}$ (文献值 ${ }^{[16]} 179 \sim 181{ }^{\circ} \mathrm{C}$ ); ${ }^{1} \mathrm{H}$ NMR $\left(\mathrm{CDCl}_{3}, 400 \mathrm{MHz}\right) \delta: 1.16 \sim 2.02(\mathrm{~m}, 10 \mathrm{H})$, $2.90 \sim 2.93(\mathrm{~m}, 1 \mathrm{H}), 3.03$ (brs, $1 \mathrm{H}), 6.78(\mathrm{t}, J=7.2 \mathrm{~Hz}$, $1 \mathrm{H}), 7.10(\mathrm{t}, J=7.2 \mathrm{~Hz}, 1 \mathrm{H}), 7.40(\mathrm{~d}, J=8.2 \mathrm{~Hz}, 2 \mathrm{H}), 7.53$ (d, $J=8.4 \mathrm{~Hz}, 1 \mathrm{H}), 7.92$ (d, $J=8.4 \mathrm{~Hz}, 2 \mathrm{H}), 8.09$ (d, $J=$ $7.2 \mathrm{~Hz}, 1 \mathrm{H}) ;{ }^{13} \mathrm{C}$ NMR $\left(\mathrm{CDCl}_{3}, 100 \mathrm{MHz}\right) \delta: 24.8,25.6$, $34.2,57.1,111.8,118.0,122.5,123.9,124.7,128.2,128.7$, 133.0, 133.3, 136.1, 141.9. Anal. calcd for $\mathrm{C}_{19} \mathrm{H}_{20} \mathrm{ClN}_{3}$ : C 70.04, H 6.19, N 12.90; found C 70.15, H 6.02, N 13.02.

$N$-叔丁基-2-(4-澳苯基)咪唑并 $[1,2-a]$ 吡啶-3-胺(4d): 褐色固体. m.p. $148 \sim 150{ }^{\circ} \mathrm{C}$ (文献值 ${ }^{[7 j]} 146{ }^{\circ} \mathrm{C}$ ); ${ }^{1} \mathrm{H}$ NMR $\left(\mathrm{CDCl}_{3}, 400 \mathrm{MHz}\right) \delta: 0.93$ (s, 9H), 2.98 (brs, 1H), $6.62 \sim 6.67(\mathrm{~m}, 1 \mathrm{H}), 7.09 \sim 7.41(\mathrm{~m}, 4 \mathrm{H}), 7.70(\mathrm{~d}, J=8.2$ $\mathrm{Hz}, 2 \mathrm{H}), 8.05(\mathrm{~d}, J=6.8 \mathrm{~Hz}, 1 \mathrm{H}) ;{ }^{13} \mathrm{C}$ NMR $\left(\mathrm{CDCl}_{3}, 100\right.$ MHz) $\delta: 29.9,56.3,111.7,117.8,122.0,123.5,123.7$, 125.1, 130.1, 131.5, 134.4, 138.0, 143.0. Anal. calcd for $\mathrm{C}_{17} \mathrm{H}_{18} \mathrm{BrN}_{3}$ : C 59.31, H 5.27, N 12.21; found C 59.21, H 5.20, N 12.08 .

$N$-环己基-2-(4-硝基苯基)咪唑并 [1,2- $a$ ] 吡啶-3-胺 (4e): 黄色固体. m.p. $236 \sim 237{ }^{\circ} \mathrm{C}$ (文献值 ${ }^{[17]} 235 \sim 237$ $\left.{ }^{\circ} \mathrm{C}\right) ;{ }^{1} \mathrm{H}$ NMR $\left(\mathrm{CDCl}_{3}, 400 \mathrm{MHz}\right) \delta: 1.02 \sim 1.79(\mathrm{~m}, 10 \mathrm{H})$, $2.86 \sim 2.89(\mathrm{~m}, 1 \mathrm{H}), 5.23$ (brs, $1 \mathrm{H}), 6.62 \sim 6.60(\mathrm{~m}, 1 \mathrm{H})$, 
$7.22 \sim 7.28(\mathrm{~m}, 1 \mathrm{H}), 7.63 \sim 7.70(\mathrm{~m}, 1 \mathrm{H}), 7.92 \sim 7.99(\mathrm{~m}$, $1 \mathrm{H}), 8.13(\mathrm{~d}, J=8.8 \mathrm{~Hz}, 2 \mathrm{H}), 8.39(\mathrm{~d}, J=8.8 \mathrm{~Hz}, 2 \mathrm{H}) ;{ }^{13} \mathrm{C}$ NMR $\left(\mathrm{CDCl}_{3}, 100 \mathrm{MHz}\right) \delta: 24.9,25.4,33.9,57.3,113.0$, $117.1,124.2,124.4,137.2,128.1,128.6,133.2,144.3$, 147.6, 153.6. Anal. calcd for $\mathrm{C}_{19} \mathrm{H}_{20} \mathrm{~N}_{4} \mathrm{O}$ : C 67.84, H 5.99, N 16.66; found C 68.03, H 6.08, N 16.53.

$N$-叔丁基-2-(3-差基苯基)咪唑并 $[1,2-a$ 吡啶-3-胺 (4f): 白色固体. m.p. $126 \sim 127{ }^{\circ} \mathrm{C}$ (文献值 ${ }^{[7 j]} 126{ }^{\circ} \mathrm{C}$ ); ${ }^{1} \mathrm{H}$ NMR $\left(\mathrm{CDCl}_{3}, 400 \mathrm{MHz}\right) \delta: 0.97$ (s, 9H), 3.56 (brs, 1H), 4.55 (brs, $1 \mathrm{H}), 6.70 \sim 6.78(\mathrm{~m}, 2 \mathrm{H}), 7.10 \sim 7.17(\mathrm{~m}, 2 \mathrm{H})$, $7.32 \sim 7.50(\mathrm{~m}, 3 \mathrm{H}), 8.28(\mathrm{~d}, J=8.4 \mathrm{~Hz}, 1 \mathrm{H}) ;{ }^{13} \mathrm{C} \mathrm{NMR}$ $\left(\mathrm{CDCl}_{3}, 100 \mathrm{MHz}\right) \delta: 28.9,55.8,111.0,113.8,113.8$, $114.7,117.9,122.7,122.8,123.6,127.8,134.9,137.6$, 140.8, 155.9. Anal. calcd for $\mathrm{C}_{17} \mathrm{H}_{19} \mathrm{~N}_{3} \mathrm{O}: \mathrm{C} 72.57$, H 6.81, N 14.94; found C 72.63, H 6.78; N 15.03.

$N$-环已基-2-(呋喃-2-基)咪唑并 [1,2- $a$ ]吡啶-3-胺 (4g): 黄色固体. m.p. $122 \sim 123{ }^{\circ} \mathrm{C}$ (文献值 ${ }^{[7 k]} 121 \sim 122$ $\left.{ }^{\circ} \mathrm{C}\right) ;{ }^{1} \mathrm{H}$ NMR $\left(\mathrm{CDCl}_{3}, 400 \mathrm{MHz}\right) \delta: 1.11 \sim 1.42(\mathrm{~m}, 5 \mathrm{H})$, $1.60 \sim 1.97(\mathrm{~m}, 5 \mathrm{H}), 2.95 \sim 3.00(\mathrm{~m}, 1 \mathrm{H}), 6.52 \sim 6.54(\mathrm{~m}$, $1 \mathrm{H}), 6.79$ (t, $J=7.2 \mathrm{~Hz}, 1 \mathrm{H}), 6.90$ (d, $J=3.2 \mathrm{~Hz}, 1 \mathrm{H}), 7.14$ $(\mathrm{t}, J=8.0 \mathrm{~Hz}, 1 \mathrm{H}), 7.48 \sim 7.52(\mathrm{~m}, 2 \mathrm{H}), 8.02(\mathrm{~d}, J=7.2$ $\mathrm{Hz}, 1 \mathrm{H}) ;{ }^{13} \mathrm{C} \mathrm{NMR}\left(\mathrm{CDCl}_{3}, 100 \mathrm{MHz}\right) \delta: 25.2,25.8,34.3$, $56.8,105.9,111.6,111.8,116.9,122.8,124.3,141.2$, 141.5, 142.6, 150.6, 160.9. Anal. calcd for $\mathrm{C}_{17} \mathrm{H}_{19} \mathrm{~N}_{3} \mathrm{O}: \mathrm{C}$ 72.57, H 6.81, N 14.94; found C 72.59, H 6.85; N 14.99.

8-甲基- $N$-环己基-2-(4-氯苯基)咪唑并 $[1,2-a$ 吡啶-3胺(4h): 褐色固体. m.p. $109 \sim 110{ }^{\circ} \mathrm{C}$ (文献值 ${ }^{[7 j]} 108{ }^{\circ} \mathrm{C}$ ); ${ }^{1} \mathrm{H}$ NMR $\left(\mathrm{CDCl}_{3}, 400 \mathrm{MHz}\right) \delta: 1.09 \sim 1.80(\mathrm{~m}, 10 \mathrm{H}), 2.68$ $(\mathrm{s}, 3 \mathrm{H}), 2.90 \sim 2.93(\mathrm{~m}, 1 \mathrm{H}), 3.10$ (brs, $1 \mathrm{H}), 6.72$ (t, $J=7.0$ $\mathrm{Hz}, 1 \mathrm{H}), 6.90 \sim 6.94(\mathrm{~m}, 1 \mathrm{H}), 7.45(\mathrm{~d}, J=7.0 \mathrm{~Hz}, 2 \mathrm{H})$, $7.92(\mathrm{~d}, J=7.0 \mathrm{~Hz}, 1 \mathrm{H}), 8.04(\mathrm{~d}, J=6.8 \mathrm{~Hz}, 2 \mathrm{H}) ;{ }^{13} \mathrm{C}$ NMR $\left(\mathrm{CDCl}_{3}, 100 \mathrm{MHz}\right) \delta: 16.5,24.8,25.6,34.0,57.2$, $111.7,121.0,123.2,124.9,127.0,128.5,128.6,133.0$, 133.6, 135.7, 143.0. Anal. calcd for $\mathrm{C}_{20} \mathrm{H}_{22} \mathrm{ClN}_{3}$ : C 70.68. H 6.52, N 12.36; found C 70.72; H 6.55, N, 12.32 .

6-甲基- $N$-叔丁基-2-(4-氯苯基)咪唑并 $[1,2-a$ ]吡啶-3胺(4i): 白色固体. m.p. $214 \sim 215{ }^{\circ} \mathrm{C}$ (文献值 ${ }^{[7 \mathrm{j}]} 215 \sim 217$ $\left.{ }^{\circ} \mathrm{C}\right) ;{ }^{1} \mathrm{H} \mathrm{NMR}\left(\mathrm{CDCl}_{3}, 400 \mathrm{MHz}\right) \delta: 0.96(\mathrm{~s}, 9 \mathrm{H}), 2.21(\mathrm{~s}$, 3H), 3.11 (brs, 1H), 6.92 (d, $J=9.0 \mathrm{~Hz}, 1 \mathrm{H}), 7.25 \sim 7.41$ (m, 4H), 7.88 (d, J=7.6 Hz, 2H), 7.99 (s, 1H); ${ }^{13} \mathrm{C}$ NMR $\left(\mathrm{CDCl}_{3}, 100 \mathrm{MHz}\right) \delta: 18.0,30.2,55.9,116.0,121.0,121.1$, $121.4,123.3,126.9,128.2,128.3,136.0,138.9,141.7$. Anal. calcd for $\mathrm{C}_{18} \mathrm{H}_{21} \mathrm{~N}_{3}$ : C 77.38, H 7.58, N 15.04; found C 77.43, H 7.55, N 14.92.

6-甲基- $N$-叔丁基-2-(3,4,5-三甲氧基苯基)咪唑并
[1,2- $a$ 吡啶-3-胺(4j): 黄色半固体(文献值 ${ }^{[7 j]}$ 黄色半固 体); ${ }^{1} \mathrm{H}$ NMR $\left(\mathrm{CDCl}_{3}, 400 \mathrm{MHz}\right) \delta: 1.02$ (s, 9H), 1.27 (brs, 1H), 2.36 (s, 3H), 3.82 (s, 3H), $3.96(\mathrm{~s}, 6 \mathrm{H}), 7.03$ (d, $J=$ $9.2 \mathrm{~Hz}, 2 \mathrm{H}), 7.48$ (d, J=8.8 Hz, 2H), $7.99(\mathrm{~s}, 1 \mathrm{H}) ;{ }^{13} \mathrm{C}$ NMR $\left(\mathrm{CDCl}_{3}, 100 \mathrm{MHz},\right) \delta: 18.2,30.1,30.8,56.6,60.8$, $106.2,115.9,121.3,121.7,122.9,127.9,130.5,137.5$, 138.3, 141.2, 152.9. Anal. calcd for $\mathrm{C}_{21} \mathrm{H}_{27} \mathrm{~N}_{3} \mathrm{O}_{3}$ : C 68.27, H 7.37, N 11.37; found C 68.20, H 7.22, N, 11.42 .

6-甲基- $N$-叔丁基-2-(4-硝基基苯基)咪唑并 $[1,2-a$ 吡 啶-3-胺(4k): 黄色固体. m.p. 231 233 ${ }^{\circ} \mathrm{C}$ (文献值 ${ }^{[7 \mathrm{j}]}$ $\left.231 \sim 232{ }^{\circ} \mathrm{C}\right)$; ${ }^{1} \mathrm{H}$ NMR $\left(\mathrm{CDCl}_{3}, 400 \mathrm{MHz}\right) \delta: 1.08(\mathrm{~s}$, 9H), 2.38 (s, 3H), 3.09 (brs, 1H), 7.04 (d, $J=8.0 \mathrm{~Hz}, 1 \mathrm{H})$, $7.44(\mathrm{~d}, J=8.8 \mathrm{~Hz}, 1 \mathrm{H}), 7.91(\mathrm{~s}, 1 \mathrm{H}), 8.17 \sim 8.24(\mathrm{~m}, 4 \mathrm{H})$; ${ }^{13} \mathrm{C} \mathrm{NMR}\left(\mathrm{CDCl}_{3}, 100 \mathrm{MHz}\right) \delta: 18.5,30.2,56.9,117.0$, $121.3,122.5,123.2,125.0,128.2,128.9,135.9,141.2$, 141.3, 147.2. Anal. calcd for $\mathrm{C}_{18} \mathrm{H}_{20} \mathrm{~N}_{4} \mathrm{O}_{2}$ : C 66.65, H 6.21, N 17.27; found C 66.75, H 6.18, N, 17.32.

6-甲基- $N$-叔丁基-2-(4-溴基苯基)咪唑并 $[1,2-a$ 吡 啶-3-胺(4l): 黄色固体. m.p. 201 203 ${ }^{\circ} \mathrm{C}$ (文献值 ${ }^{[7 \mathrm{j}]} 203$ $\left.{ }^{\circ} \mathrm{C}\right) ;{ }^{1} \mathrm{H}$ NMR $\left(\mathrm{CDCl}_{3}, 400 \mathrm{MHz}\right) \delta: 1.06$ (s, 9H), 1.28 (bs, 1H), 2.38 (s, 3H), 6.99 (d, $J=9.2 \mathrm{~Hz}, 1 \mathrm{H}), 7.47$ (d, $J=8.8$ $\mathrm{Hz}, 1 \mathrm{H}), 7.57$ (d, $J=8.4 \mathrm{~Hz}, 2 \mathrm{H}), 7.88$ (d, $J=8.8 \mathrm{~Hz}, 2 \mathrm{H})$, $7.98(\mathrm{~s}, 1 \mathrm{H}) ;{ }^{13} \mathrm{C} \mathrm{NMR}\left(\mathrm{CDCl}_{3}, 100 \mathrm{MHz}\right) \delta: 17.8,30.1$, $55.8,115.6,120.2,120.5,120.6,122.84,126.9,128.2$, $129.9,132.0,135.8,140.1$. Anal. calcd for $\mathrm{C}_{18} \mathrm{H}_{20} \mathrm{BrN}_{3}$ : C 60.34, H 5.63, N 11.73; found C 60.11, H 5.56, N 11.62.

6-甲基- $N$-环己基-2-(噻吩-2-基)咪唑并 [1,2- $a$ 吡 啶-3-胺 (4m)：黄色固体. m.p. 200 201 ${ }^{\circ} \mathrm{C}$ (文献值 ${ }^{[7 \mathrm{k}]}$ $\left.203{ }^{\circ} \mathrm{C}\right) ;{ }^{1} \mathrm{H} \mathrm{NMR}\left(\mathrm{CDCl}_{3}, 400 \mathrm{MHz}\right) \delta: 1.20 \sim 1.90(\mathrm{~m}$, $\left.10 \mathrm{H}_{1}\right), 2.38(\mathrm{~s}, 3 \mathrm{H}), 3.08(\mathrm{brs}, 1 \mathrm{H}), 6.99 \sim 7.68(\mathrm{~m}, 5 \mathrm{H})$, $7.88(\mathrm{~s}, 1 \mathrm{H}) ;{ }^{13} \mathrm{C} \mathrm{NMR}\left(\mathrm{CDCl}_{3}, 100 \mathrm{MHz}\right) \delta: 18.6,24.9$, 26.1, 34.3, 57.1, 57.2, 116.4, 120.6, 121.3, 124.0, 124.6, $126.0,127.78,128.5,132.7,137.2,141.2$. Anal. calcd for $\mathrm{C}_{18} \mathrm{H}_{21} \mathrm{~N}_{3} \mathrm{~S}$ : C 69.42, H 6.80, N 13.49, S 10.30; found $\mathrm{C}$ 69.28, H 6.86, N 13.40, S 10.09 .

\section{References}

[1] (a) Sasaki, A.; Kitamura, K.; Alcalde, R. E.; Tanaka, T.; Suzuki, A.; Etoh, Y.; Matsumura, T. Int. J. Caner 1998, 77, 279.

(b) Jaramillo, C.; de Diego, J. E.; Hamdouchi, C.; Collins, E.; Keyser, H.; Sánchez-Martínez, C.; del Prado, M.; Norman, B.; Brooks, H. B.; Watkins, S. A.; Spencer, C. D.; Dempsey, J. A.; Anderson, B. D.; Campbell, R. M.; Leggett, T.; Patel, B.; Schultz, R. M.; Espinosa, J.; Vieth, M.; Zhang, F.; Timm, D. E. Bioorg. Med. Chem. Lett. 2004, 20, 6095.

(c) Hayakawa, M.; Kaizawa, H.; Kawaguchi, K.; Ishikawa, N.; Koizumi, T.; Ohishi, T.; Yamano, M.; Okada, M.; Ohta, M.; Tsukamoto, S.; Raynaud, F. I.; Waterfield, M. D.; Parker, P.; Workman, 
P. Bioorg. Med. Chem. 2007, 15, 403.

[2] (a) Gudmundsson, K. S.; Williams, J. D.; Drach, J. C.; Townsend, L. B. J. Med. Chem. 2003, 46, 1449.

(b) Hamdouchi, C.; de Blas, J.; del Prado, M.; Gruber, J.; Heinz, B. A.; Vance, L. J. Med. Chem. 1999, 42, 50.

(c) Lhassani, M.; Chavignon, O.; Chezal, J. M.; Teulade, J. C.; Chapat, J. P.; Snoeck, R.; Andrei, G.; Balzarini, J.; Clercq, E.; Gueier, A. Eur. J. Med. Chem. 1999, 34, 271.

[3] Starrett, J. E.; Montzka, T. A.; Crosswell, A. R.; Cavanagh, R. L. J. Med. Chem. 1989, 32, 2204.

[4] Kercher, T.; Rao, C.; Bencsik, J. R.; Josey, J. A. J. Comb. Chem. 2007, 9, 1177.

[5] Ismail, M. A.; Brun, R.; Wenzler, T.; Tanious, F. A.; Wilson, W. D.; Boykin, D. W. J. Med. Chem. 2004, 47, 3658.

[6] (a) Zhou, J. L.; Liu, J. C.; Chen, Q. Y. Chin. J. Org. Chem. 2009, 29, 1708 (in Chinese).

(周建良, 刘建超, 陈启元, 有机化学, 2009, 29, 1708.)

(b) Zhang, R. Ph.D. Dissertation, Zhejiang University, Hangzhou, 2007 (in Chinese).

(张睿, 博士论文, 浙江大学, 杭州, 2007.)

(c) Li, Z. R. M.S. Thesis, Qingdao University of Science and Technology, Qingdao, 2009 (in Chinese).

(李兆瑞，硕士论文，青岛科技大学，杭州, 2009.)

[7] (a) Chen, J. J.; Golebiowski, A.; McClenaghan, J.; Klopfenstein, S.

R.; West, L. Tetrahedron Lett. 2001, 2269.

(b) Lyon, M. A.; Kercher, T. S. Org. Lett. 2004, 6, 4989.

(c) Blackburn, C.; Guan, B. Tetrahedron Lett. 2000, 41, 1495.

(d) Rostamnia, S.; Lamei, K.; Mohammadquli, M.; Sheykhan, M.; Heydari, A. Tetrahedron Lett. 2012, 53, 5257.

(e) Bienayme, H.; Bouzid, K. Angew. Chem., Int. Ed. 1998, 37, 2234.

(f) Shaabani, A.; Soleimani, E.; Maleki, A.; Moghimi-Rad, J. Synth. Commun. 2008, 38, 1090.

(g) Shaabani, A.; Maleki, A.; Rad, J.; Soleimani, E. Chem. Pharm. Bull. 2007, 55, 957.

(h) Rousseau, A. L.; Matlaba, P.; Parkinson, C. J. Tetrahedron Lett. 2007, 48, 4079.

(i) Bode, M. L.; Gravestock, D.; Moleele, S. S.; vander Westhuyzen
C. W.; Pelly, S. C.; Steenkamp, P. A.; Hoppe, H. C.; Khan, T.; Nkabinde, L. A. Bioorg. Med. Chem. 2011, 19, 4227.

(j) Khan, A. T.; Basha, R. S.; Lal, M. Tetrahedron Lett. 2012, 53, 2211.

(k) Shaabani, A.; Soleimani, E.; Sarvari, A.; Rezayan, A. H.; Maleki, A. Chin. J. Chem. 2009, 27, 369.

[8] (a) Blackburn, G. B.; Fleming, P.; Shiosaki, K.; Tsai, S. Tetrahedron Lett. 1998, 39, 3635.

(b) Shaabani, A.; Soleimani, E.; Sarvary, A.; Rezayan, A. H.; Maleki, A. Chin. J. Chem. 2009, 27, 369.

(c) Odell, L. R.; Nilsson, M.; Gising, J.; Lagerlund, O.; Muthas, D.; Nordqvist, A.; Karlen, A.; Larhed, M. Bioorg. Med. Chem. Lett. 2009, 19, 4790 .

(d) Rousseau, A. L.; Matlaba, P.; Parkinson, C. J. Tetrahedron Lett. 2007, 48, 4079.

(e) Shaabani, A.; Soleimani, E.; Maleki, A.; Moghimi-Rad, J. Synth. Commun. 2008, 38, 1090.

(f) Shukla, N. M.; Salunke, D. B.; Yoo, E.; Mutz, C. A.; Balakrishna, R.; David, S. A. Bioorg. Med. Chem. 2012, 20, 5850.

(g) Shaabani, A.; Maleki, A.; Moghimi, R. J.; Soleimani, E. Chem. Pharm. Bull. 2007, 55, 957.

[9] (a) Varma, R. S.; Kumar, D. Tetrahedron Lett. 1999, 40, 7665.

(b) Ireland, S. M.; Tye, H.; Whittaker, M. Tetrahedron Lett. 2003, 44, 4369.

[10] Shaabani, A.; Soleimani, E.; Maleki, A. Tetrahedron Lett. 2006, 47, 3031.

[11] Yang, C. G.; Fang, L. Z.; Wu, L. Q.; Yan, F. L. Asian J. Chem. 2010, $22,6031$.

[12] Sharma, G. V. M.; Reddy, J. J.; Lakshmi, P. S.; Krishna, P. R. Tetrahedron Lett. 2004, 45, 7729.

[13] Wu, L. Q.; Yang, X. J.; Wang, X.; Yan, F. L. J. Sulfur Chem. 2010, 31,509 .

[14] Bigdeli, M. A.; Heravi, M. M.; Mahdavinia, G. H. Catal. Commun. 2007, 8, 1595.

[15] Sun, P. L.; Fang, L. Z.; Wu, L. Q. J. Sulfur Chem. 2011, 32, 257.

[16] Adib, M.; Mahdavi, M.; Noghani, M. A.; Mirzaei, P. Tetrahedron Lett. 2007, 48, 7263.

[17] Adib, M.; Sheikhi, E.; Rezaei, N. Tetrahedron Lett. 2011, 52, 3191.

(Qin, X.) 